Paper

\title{
Efficient Acquisition of Light Transport Matrix Using Push Broom-Type Projector Illumination
}

\author{
Isao Miyagawa (member) $^{\dagger}$, Hiroyuki Arai (member) ${ }^{\dagger}$, Yukinobu Taniguchi (member) ${ }^{\dagger}$
}

\begin{abstract}
We propose a practical method that efficiently acquires light transports from arbitrary objects by using push broom-type projector illumination; vertical-strip and horizontal-strip light patterns are used. The combinations of verticalstrip and horizontal-strip camera responses allow our method to obtain light transports between each overlapped projector pixel and the camera response detected in common. Our method significantly reduces the number of camera response images needed for acquiring all light transports compared to brute-force scanning. Tests of the proposed method using real images show that it obtains more accurate light transports from various objects than brute-force scanning, and the resulting light transport matrix faithfully reproduces the original images. We demonstrate a relighting test based on the light transport matrix and confirm that our method yields relighted images that closely approximate the actual images captured by a camera.
\end{abstract}

Key words: light transport matrix, projector illumination, relighting, color mixture, projector-camera system

\section{Introduction}

Projection mapping provides seamless image displays with excellent presence so viewers can more fully appreciate the video art projected into actual environments. Conventional approaches demand the design of a model to simulate the interaction of the projection-based displays with the actual 3D structures, as well as timeconsuming trial and error adjustments of the system parameters or CG contents to suit the models. Given the difficulty of measuring the 3D structures and calibrating projection-based display systems, simulations using the designed model are neither quick nor easy from the viewpoint of plug-and-go integration. What is needed is a projection system that has no complicated design or setup phase but well supports CG creators and general operators.

If we want to use an uncalibrated projector-camera system to display an arbitrary image onto a target that has unknown 3D structures, we need the light transports $^{1) \sim 5)}$; they represent the geometric and photometric correspondences between projector and camera pixels. Sequentially lighting each pixel on a projector plane by brute-force scanning (BFS) yields various direct and indirect reflected lights that are captured by a cam$\mathrm{era}^{1) 2)}$. We can use the light transport matrix (LTM) to

Received June 13, 2014; Revised August 29, 2014; Accepted October 6,2014

$\dagger$ NTT Media Intelligence Laboratories, NTT Corporation (1-1 Hikari-no-oka Yokosuka Kanagawa 239-0847, Japan) handle these light transports simultaneously; the LTM column vector represents the camera image that corresponds to each projector light. It has been found that LTM is useful for relighting in $\mathrm{CG}^{236)}$, projectionbased AR, and the inverse matrix supports radiometric compensation to display desired images on 3D structures $^{4) 7}$. Unfortunately, BFS is not realistic because it needs to capture camera response images whose number dramatically explodes with the number of projector pixels. On the assumption that most LTM are sparse high-rank matrices, some solutions create a projector illumination pattern with both high and low frequencies to acquire LTM from as few camera response images as possible ${ }^{2) 8}$. For instance, the acquisition method in 2) demands scene-independent ensembles to be designed for the illumination patterns, and the method developed in 8) uses gray code patterns and multiplexed illumination patterns, simultaneously. In contrast, approaches that handle dense low-rank matrices assume that high frequency projector lights are seldom present in the camera responses, i.e. low-rank matrix approximation $^{9) \sim 11)}$. If LTM has the properties of dense and low-rank, calculating the inverse matrix is very tractable. However, the low-rank matrix approximation demands a special projector-camera system with optical apparatus and a diffuser to acquire the dense low-rank LTM; these preconditions greatly narrow the scope of LTM application.

From a practical standpoint, we assume that camera responses contain direct and indirect reflected lights 
with various frequencies as with BFS. In this paper, we aim to realize a practical method that efficiently acquires all light transports from arbitrary complex objects. Most existing methods acquire light transports by using white illumination ${ }^{2) 8)}{ }^{10)}$. As projector and camera generally exhibit inherent spectra in the visible light region, we consider color mixture components, socalled crosstalk between the projector's and camera's channels, in projector-camera systems ${ }^{12)}$. In Section 2, we introduce a new LTM that handles the color mixing of projector and camera pixels; in matrix format, direct and indirect reflected lights are handled by extending the color-mixing matrix ${ }^{13) \sim 15)}$. We describe the LTM by using a set of projector illuminations and camera responses. In Section 3, we employ push broom-type projector illumination, which consists of multiple vertical-strip and horizontal-strip patterns for each channel. Compared to previous works that handle sparse high-rank LTM, the projector illumination is simply and automatically generated in uncalibrated projector-camera systems without requiring the design of appropriate illumination patterns. We sequentially employ the illumination patterns to acquire the LTM that has the color mixture components. By utilizing all combinations of the vertical-strip and horizontal-strip camera responses, our method obtains light transports between the overlapped projector pixel and the camera response. Our method uses significantly fewer camera response images to acquire the whole LTM compared to BFS. Section 4 tests both BFS and our method for acquiring LTM of various objects, and the results show that our method acquires complex light transports more accurately than BFS. We demonstrate that our method yields good relighting images that are close to the actual images captured by a camera. Finally, Section 5 concludes this study and mentions future work.

\section{Extended-LTM}

We assume that the camera plane has $M \times M$ pixels and the projector plane has $N \times N$ pixels for convenience. By considering crosstalk between the projector's and camera's channels, we define a $3 M^{2} \times 3 N^{2}$ matrix

$$
\mathbf{T}=\left[\begin{array}{ccc}
\mathbf{T}_{r r} & \mathbf{T}_{r g} & \mathbf{T}_{r b} \\
\mathbf{T}_{g r} & \mathbf{T}_{g g} & \mathbf{T}_{g b} \\
\mathbf{T}_{b r} & \mathbf{T}_{b g} & \mathbf{T}_{b b}
\end{array}\right]
$$

where sub-matrix $\mathbf{T}_{\alpha \beta}, \alpha, \beta \in\{r, g, b\}$ represents $M^{2} \times$ $N^{2}$ LTM that transports projector pixels of $\beta$-channel

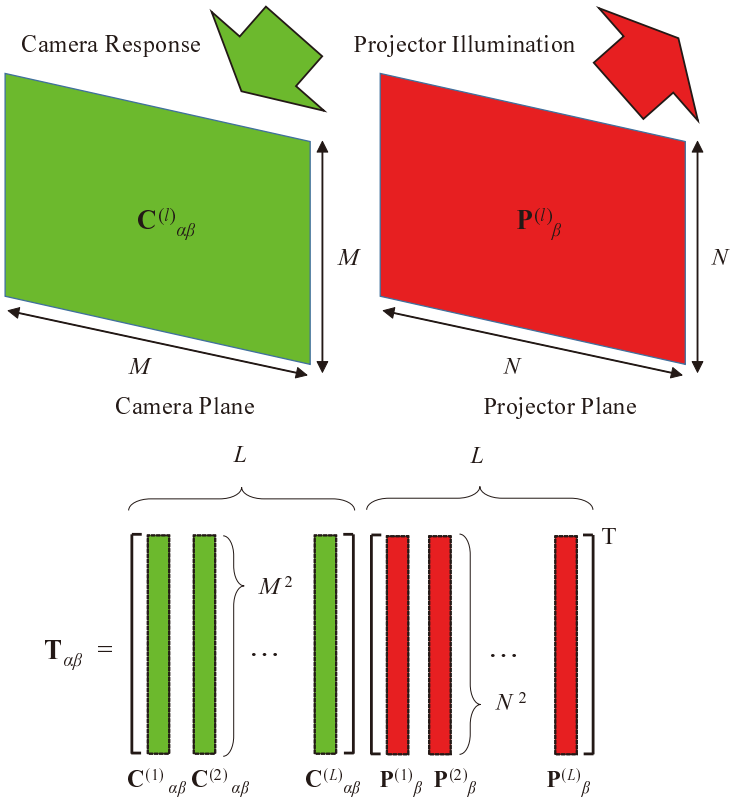

Fig. 1 Acquisition of sub-matrix $\mathbf{T}_{\alpha \beta}$.

to camera pixels of $\alpha$-channel. In this paper, we call matrix $\mathbf{T}$ the extended-LTM.

Based on vectorization of a color image input to the projector, we denote the projector illumination by the $3 N^{2}$-dimensional vector $\left[\mathbf{P}_{r}^{T}, \mathbf{P}_{g}^{T}, \mathbf{P}_{b}^{T}\right]^{T}$. The R-, G-, and B-channel's projector illuminations correspond to $\mathbf{P}_{r}, \mathbf{P}_{g}$, and $\mathbf{P}_{b}$, respectively. Similarly, we assume that camera response is given by the $3 M^{2}$-dimensional vector $\left[\mathbf{C}_{r}^{T}, \mathbf{C}_{g}^{T}, \mathbf{C}_{b}^{T}\right]^{T}$, where $\mathbf{C}_{r}, \mathbf{C}_{g}$, and $\mathbf{C}_{b}$ correspond to camera responses of each channel. We then describe the geometric and photometric correspondence between the projector pixels and the camera pixels,

$$
\left[\begin{array}{c}
\mathbf{C}_{r} \\
\mathbf{C}_{g} \\
\mathbf{C}_{b}
\end{array}\right]=\mathbf{T}\left[\begin{array}{c}
\mathbf{P}_{r} \\
\mathbf{P}_{g} \\
\mathbf{P}_{b}
\end{array}\right]+\mathbf{F},
$$

by using the extended-LTM. Vector $\mathbf{F}$ represents environmental light, which includes black offsets from the projector, as detected by the camera response. The color-mixing matrix ${ }^{13) \sim 15)}$ generally describes the photometric input/output correspondence, and indicates direct reflection based on one-to-one mapping between projector and camera pixels, in a calibrated projectorcamera system. In contrast, extended-LTM describes both geometric and photometric input/output correspondence in an uncalibrated projector-camera system in order to handle direct and indirect reflections between projector pixels and camera pixels.

Fig. 1 illustrates how to acquire sub-matrix $\mathbf{T}_{\alpha \beta}$. The system outputs $L$-kinds of projector illuminations and detects the camera responses, one after the other. 
Here, we denote the $l$-th projector illumination of $\beta$ channel by the $N^{2}$-dimensional vector $\mathbf{P}_{\beta}^{(l)}$, and the $l$ th camera response of $\alpha$-channel connected with the $\beta$-channel of the $l$-th projector illumination by the $M^{2}$ dimensional vector $\mathbf{C}_{\alpha \beta}^{(l)}$. In general, we can describe sub-matrix $\mathbf{T}_{\alpha \beta}$ as follows,

$$
\begin{aligned}
\mathbf{T}_{\alpha \beta} & =\hat{\mathbf{C}}_{\alpha \beta} \hat{\mathbf{P}}_{\beta}^{T}, \\
\hat{\mathbf{C}}_{\alpha \beta} & =\left[\begin{array}{llll}
\mathbf{C}_{\alpha \beta}^{(1)} & \mathbf{C}_{\alpha \beta}^{(2)} & \cdots & \mathbf{C}_{\alpha \beta}^{(L)}
\end{array}\right], \\
\hat{\mathbf{P}}_{\beta} & =\left[\begin{array}{llll}
\mathbf{P}_{\beta}^{(1)} & \mathbf{P}_{\beta}^{(2)} & \cdots & \mathbf{P}_{\beta}^{(L)}
\end{array}\right],
\end{aligned}
$$

by using $L$-kinds of projector illuminations and the corresponding camera responses * The $l$-th column vector of $M^{2} \times L$ matrix $\hat{\mathbf{C}}_{\alpha \beta}$ corresponds to $\mathbf{C}_{\alpha \beta}^{(l)}$ and the $l$ th column vector of $N^{2} \times L$ matrix $\hat{\mathbf{P}}_{\beta}$ corresponds to $\mathbf{P}_{\beta}^{(l)}$. As shown in Eqs. (4) and (5), sub-matrix $\mathbf{T}_{\alpha \beta}$ preserves camera vector $\mathbf{C}_{\alpha \beta}^{(l)}$ and projector vector $\mathbf{P}_{\beta}^{(l)}$ in sequential order.

By multiplying Eq. (3) by the $m$-th projector vector $\mathbf{P}_{\beta}^{(m)}$, we obtain the following equation,

$$
\mathbf{T}_{\alpha \beta} \mathbf{P}_{\beta}^{(m)}=\left[\begin{array}{llll}
\mathbf{C}_{\alpha \beta}^{(1)} & \mathbf{C}_{\alpha \beta}^{(2)} & \cdots & \mathbf{C}_{\alpha \beta}^{(L)}
\end{array}\right]\left[\begin{array}{c}
\left(\mathbf{P}_{\beta}^{(1)}\right)^{T} \mathbf{P}_{\beta}^{(m)} \\
\left(\mathbf{P}_{\beta}^{(2)}\right)^{T} \mathbf{P}_{\beta}^{(m)} \\
\vdots \\
\left(\mathbf{P}_{\beta}^{(L)}\right)^{T} \mathbf{P}_{\beta}^{(m)}
\end{array}\right]
$$

If vectors $\mathbf{P}_{\beta}^{(l)}, l \in\{1,2, \cdots, L\}$ span an orthonormal system, we have

$$
\left(\mathbf{P}^{(l)}\right)^{T} \mathbf{P}_{\beta}^{(m)}=\delta_{l m},
$$

where $\delta_{l m}$ indicates Kronecker delta. Eqs. (6) and (7) ensure that

$$
\mathbf{C}_{\alpha \beta}^{(l)}=\mathbf{T}_{\alpha \beta} \mathbf{P}_{\beta}^{(l)},
$$

which is equivalent to the definition of LTM, for all vectors $\mathbf{P}_{\beta}^{(l)}, \forall l$. For BFS with a single pixel light, all of the diagonal elements in matrix $\hat{\mathbf{P}}_{\beta}$ are unity, i.e. matrix $\hat{\mathbf{P}}_{\beta}$ is an $N^{2} \times N^{2}$ identity matrix. Since we obtain each camera response $\mathbf{C}_{\alpha \beta}^{(l)}$ by capturing projector illumination $\mathbf{P}_{\beta}^{(l)}$, acquisition of LTM is virtually equal to designing projector illuminations $\mathbf{P}_{\beta}^{(l)}, l \in\{1,2, \cdots, L\}$.

\section{Proposed Method}

\section{1 Push Broom-Type Illumination}

In order to acquire LTM from the fewest possible camera response images, we adopt two types of push

\footnotetext{
* The method proposed in 11) uses a set of projector illuminations
} that span Krylov subspace and the camera responses.

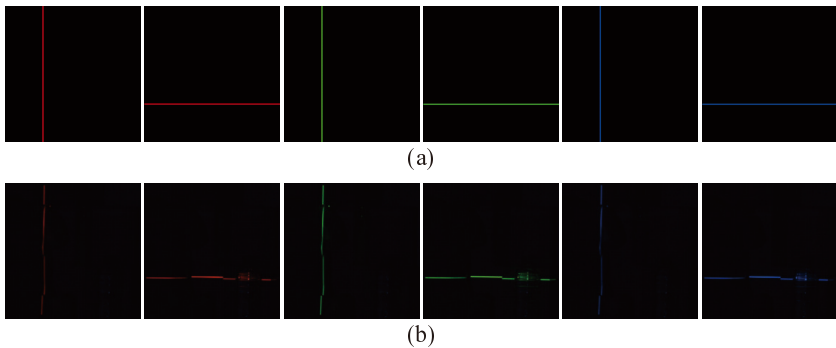

Fig. 2 Examples of projector illumination and camera response. (a) $\mathbf{Q}_{\beta}^{(i)}$ and $\mathbf{R}_{\beta}^{(j)}$. (b) $\mathbf{V}_{\alpha \beta}^{(i)}$ and $\mathbf{H}_{\alpha \beta}^{(j)}$.

broom-type projector illumination patterns. One push broom pattern moves horizontally from left to right, and the other moves vertically from bottom to top. We call the former vertical-strip illumination and the latter horizontal-strip illumination for convenience of explanation. Given $N \times N$ projector pixels in the system, we divide the projector plane into multiple vertical-strip illuminations that have $n \times N$ pixels, and horizontal-strip illuminations that have $N \times n$ pixels, where $n L=N^{* *}$. If both types have neither gap nor overlap, the system has $2 L$-kinds of projector illuminations.

Assume that the projector outputs the $i$-th verticalstrip illumination pattern, $i \in\{1,2, \cdots, L\}$, in sequence. We obtain the $N^{2}$-dimensional projector vector $\mathbf{Q}_{\beta}^{(i)}$ by the vectorization and normalization of the projector illumination. The projector vectors $\mathbf{Q}_{\beta}^{(i)}$ satisfy a normalized orthogonal system, i.e. Eq. (7). Similarly, when the projector outputs the $j$-th horizontal-strip illumination pattern in sequence, the projector vectors $\mathbf{R}_{\beta}^{(j)}, j \in\{1,2, \cdots, L\}$, also satisfy a normalized orthogonal system.

\section{2 Acquisition of Extended-LTM}

Fig. 2 shows examples of the vertical/horizontal push broom-type projector illuminations with $N=512$ and $n=4$; camera response is detected from area $\mathrm{E}$ in Fig. 5(a). Note that vertical-strip vector $\mathbf{Q}_{\beta}^{(i)}$ and horizontal-strip vector $\mathbf{R}_{\beta}^{(j)}$ are not orthogonal so that $\left(\mathbf{Q}_{\beta}^{(i)}\right)^{T} \mathbf{R}_{\beta}^{(j)} \neq 0$. In other words, overlapped pixels on the projector plane are always $n \times n$. Our idea is to obtain light transports by using these overlapped pixels between the two illumination vectors. We denote the camera response vectors that correspond to $i$-th vertical-strip illumination $\mathbf{Q}_{\beta}^{(i)}$ and $j$-th horizontal-strip illumination $\mathbf{R}_{\beta}^{(j)}$ by $\mathbf{V}_{\alpha \beta}^{(i)}$ and $\mathbf{H}_{\alpha \beta}^{(j)}$, respectively. Assuming that sub-matrix $\mathbf{T}_{\alpha \beta}$ is unknown, we set

\footnotetext{
** When we use $n=1$ for vertical/horizontal-strip illuminations, the camera response may not detect light transports accurately due to the weak strip lights. Thus, a suitable value of $n$ should be determined as part of the setup phase.
} 


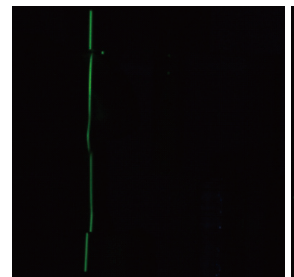

(a)

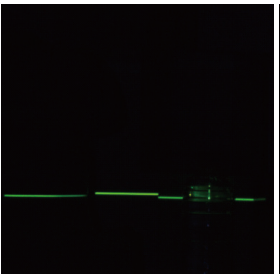

(b)

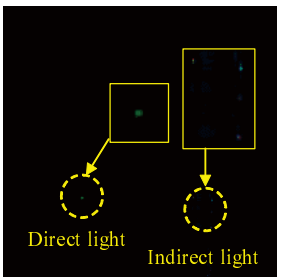

(c)
Fig. 3 Example of logical product $\mathbf{V}_{\alpha \beta}^{(i)}$ and $\mathbf{H}_{\alpha \beta}^{(j)}$. (a) $\mathbf{V}_{\alpha \beta}^{(i)}$. (b) $\mathbf{H}_{\alpha \beta}^{(j)}$. (c) $\mathbf{V}_{\alpha \beta}^{(i)} \otimes \mathbf{H}_{\alpha \beta}^{(j)}$.

$$
\begin{aligned}
\mathbf{V}_{\alpha \beta}^{(i)} & =\mathbf{T}_{\alpha \beta} \mathbf{Q}_{\beta}^{(i)} \\
\mathbf{H}_{\alpha \beta}^{(j)} & =\mathbf{T}_{\alpha \beta} \mathbf{R}_{\beta}^{(j)}
\end{aligned}
$$

according to Eq. (8).

From Eqs. (9) and (10), we obtain, in common, the camera response pixels that correspond to the overlapped $n \times n$ projector pixels ${ }^{16)}$. When width $n$ is sufficiently small, we assume that the logical product between camera response vectors $\mathbf{V}_{\alpha \beta}^{(i)}$ and $\mathbf{H}_{\alpha \beta}^{(j)}$ yields

$$
\mathbf{V}_{\alpha \beta}^{(i)} \otimes \mathbf{H}_{\alpha \beta}^{(j)}=\mathbf{T}_{\alpha \beta}\left(\frac{\mathbf{Q}_{\beta}^{(i)} \otimes \mathbf{R}_{\beta}^{(j)}}{\left\|\mathbf{Q}_{\beta}^{(i)} \otimes \mathbf{R}_{\beta}^{(j)}\right\|}\right),
$$

where symbol $\otimes$ means the logical product in terms of vector components; the concrete calculation yields the average value of two non-zero components. Eq. (11) exhibits light transports between the small square light source $\mathbf{Q}_{\beta}^{(i)} \otimes \mathbf{R}_{\beta}^{(j)}$ with $n \times n$ pixels and camera response $\mathbf{V}_{\alpha \beta}^{(i)} \otimes \mathbf{H}_{\alpha \beta}^{(j)}$. Fig. 3 shows an example of the logical product $\mathbf{V}_{\alpha \beta}^{(i)} \otimes \mathbf{H}_{\alpha \beta}^{(j)},(\beta=g)$ computed from the vertical/horizontal-strip camera responses in Fig. 2. The logical product extracts not only the direct light reflected from the gypsum torso but also indirect light reflected from the plastic bottle; see Fig. 5(a). Thus, our method obtains sub-matrix $\mathbf{T}_{\alpha \beta}$ by utilizing all combinations of vertical-strip response vectors $\mathbf{V}_{\alpha \beta}^{(i)}, \forall i$ and horizontal-strip response vectors $\mathbf{H}_{\alpha \beta}^{(j)}, \forall j$.

Push broom-type projector illumination is practical as demonstrated by existing techniques. In a catadioptric projector system ${ }^{4}$, the vertical and horizontal push broom-type illuminations reflected from a convexcurved mirror surface are used for planar displays. Assuming that each camera pixel responds to only one projector pixel, the system mainly obtains direct light transports from the combinations of vertical and horizontal camera response images. The relighting method developed in 6) uses multiple square patterns with regular spacing intervals that lie along vertical and horizontal directions. The method supposes that each square pattern yields local indirect reflections and ex- tracts in common camera responses that correspond to the square pattern that vertical and horizontal illuminations share. However, it cannot handle concaveshape objects, e.g. the bowl in Fig. 5(b), because they have complex indirect reflection lights. In contrast, our method finds not only direct light transport that correspond to each small square pattern, but also various indirect light transport from 3D objects, which are not restricted to local indirect reflections, by computing the logical product of vertical/horizontal-strip camera responses.

\subsection{Algorithm}

We summarize here an algorithm that acquires extended-LTM by using push broom-type illumination:

(i) Segment the projector plane by vertical-strip and horizontal-strip patterns, and obtain projector vectors $\mathbf{Q}_{\beta}^{(i)}, \mathbf{R}_{\beta}^{(j)}, i, j \in\{1,2, \cdots, L\}$ of $\beta$-channel.

(ii) Capture camera images by lighting projector vectors $\mathbf{Q}_{\beta}^{(i)}$ and $\mathbf{R}_{\beta}^{(j)}$ in sequence.

(iii) Obtain camera response vectors $\mathbf{V}_{\alpha \beta}^{(i)}$ and $\mathbf{H}_{\alpha \beta}^{(j)}, \alpha \in\{r, g, b\}$ from the camera responses detected by background subtraction.

(iv) Repeat steps (i) (iii) for individual projector channels $\beta \in\{r, g, b\}$, and temporarily store all vertical- and horizontal-strip projector illumination vectors and the camera response vectors.

(v) Compute the renew camera response vector,

$$
\mathbf{C}_{\alpha \beta}^{(k)}=\mathbf{V}_{\alpha \beta}^{(i)} \otimes \mathbf{H}_{\alpha \beta}^{(j)},
$$

from all vectors $\mathbf{V}_{\alpha \beta}^{(i)}$ and $\mathbf{H}_{\alpha \beta}^{(j)}$, and the renew normalized projector illumination vector,

$$
\mathbf{P}_{\beta}^{(k)}=\frac{\mathbf{Q}_{\beta}^{(i)} \otimes \mathbf{R}_{\beta}^{(j)}}{\left\|\mathbf{Q}_{\beta}^{(i)} \otimes \mathbf{R}_{\beta}^{(j)}\right\|},
$$

where $k=(j-1) L+i$.

(vi) Construct the $3 M^{2} \times 3 L^{2}$ camera response matrix,

$$
\hat{\mathbf{C}}=\left[\begin{array}{lll}
\hat{\mathbf{C}}_{r r} & \hat{\mathbf{C}}_{r g} & \hat{\mathbf{C}}_{r b} \\
\hat{\mathbf{C}}_{g r} & \hat{\mathbf{C}}_{g g} & \hat{\mathbf{C}}_{g b} \\
\hat{\mathbf{C}}_{b r} & \hat{\mathbf{C}}_{b g} & \hat{\mathbf{C}}_{b b}
\end{array}\right]
$$

using Eq. (4), and obtain the $3 N^{2} \times 3 L^{2}$ projector illumination matrix; its column vectors span an orthonormal system,

$$
\hat{\mathbf{P}}=\left[\begin{array}{lll}
\hat{\mathbf{P}}_{r} & \hat{\mathbf{P}}_{g} & \hat{\mathbf{P}}_{b}
\end{array}\right],
$$

using Eq. (5). 


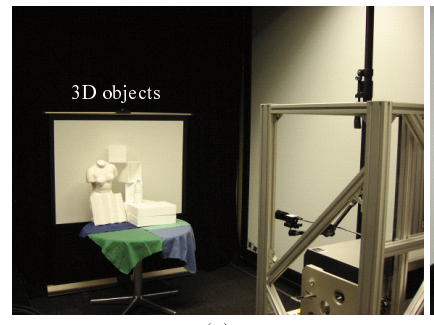

(a)

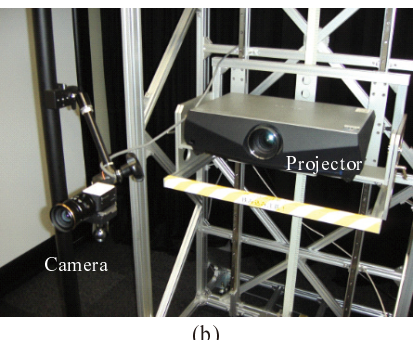

(b)
Fig. 4 Experimental system. (a) Setup. (b) Projector and camera.

Matrix multiplication $\hat{\mathbf{C}} \hat{\mathbf{P}}^{T}$ yields the extended-LTM $\mathbf{T}$, which virtually equals that acquired by BFS with $n \times n$ pixels. If BFS with $n \times n$ pixels is employed to obtain the extended-LTM, $3 L^{2}$ projector illuminations and $3 L^{2}$ camera images will be needed. As the proposed method uses $2 L$-kinds of push broom-type illuminations for each projector channel, it needs $6 L$ camera images to acquire all light transports. Note that our method reconstructs the extended-LTM from $2 / L$-th of camera response images needed by BFS with $n \times n$ pixels. Thus, our method can efficiently acquire the extended-LTM by applying push broom-type projector illumination as $L$ increases.

\section{Experiments and Results}

\section{1 Experimental System Setup}

Fig. 4 shows our experimental system setup; it consisted of an LCD projector (SONY VPL-F-41) and an IEEE1394b camera (Point Grey GRAS-50S5C-C) controlled by separate computers. The projector has a resolution of $1,600 \times 1,200$ pixels, and the camera captures one full color image per second, with a resolution of $1,600 \times 1,200$ pixels. Once the system captures an image from the camera, it transfers the observed image to the projector via LAN. Fig. 5 shows that the $3 \mathrm{D}$ objects consisted of plastic boxes, a gypsum torso, an accordion folded paper, a plastic bottle with water, a wineglass, and a stainless steel bowl.

We implemented our algorithm on a computer, OS: Windows 7 (32-bit), CPU: Intel Xeon E5520 (2.26 $\mathrm{GHz}$ ), Memory: $3.43 \mathrm{~GB}$, connected to the projector, and acquired the extended-LTM by using the push broom-type projector illuminations. We allocated working memory for camera response matrix $\hat{\mathbf{C}}$ and projector illumination matrix $\hat{\mathbf{P}}$ by employing the spalloc function implemented in MATLAB. We set pixel value 10 as a threshold in order to detect each channel's response against image noise. When we measured light

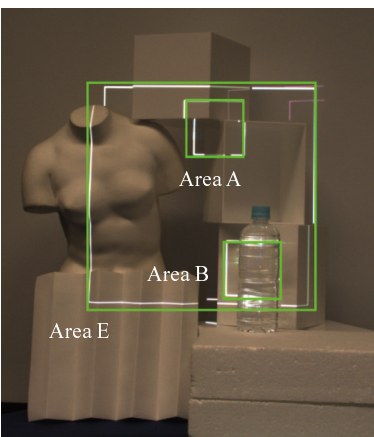

(a)

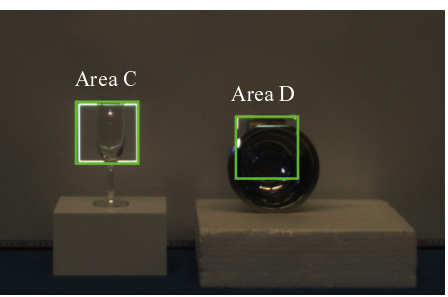

(b)
Fig. 5 Examples of 3D objects used in our experiments. (a) Scene 1. (b) Scene 2.

transports by using BFS with single pixel in a preliminary experiment, our system did not detect camera response at all due to the weak illumination light. We confirmed that it detects camera responses with no defective pixel in the environment when we used BFS with $4 \times 4$ pixels ${ }^{*}$. In subsequent experiments, we empirically set the vertical/horizontal-strip light source to yield $n=4$ pixels.

\section{2 Visualization of Extended-LTM}

We compare the extended-LTM acquired by BFS and that by our method in areas A, B, C, and D, which include some plastic boxes, a plastic bottle containing drinking water, a wineglass, and a stainless steel bowl, respectively. We marked the range of $128 \times 128$ pixels used as push broom-type projector illumination by the white frame, and the range of $128 \times 128$ pixels detected as camera response by the green frame in Fig. 5. BFS needed 1,024 camera response images per color channel to measure the extended-LTM while our method acquired it from just 64 camera response images per color channel. In Fig. 6, Fig. 7, Fig. 8, and Fig. 9, we visualize the matrix elements of $\mathbf{T}_{\alpha \beta}$ in each extendedLTM by using the mixed colors of camera channel $(\alpha)$ and projector channel $(\beta)$. These figures provide an overview of all light transports, in which each illumination source with $4 \times 4$ pixels is converted into point light source.

We ensured that both methods can capture direct reflected light and indirect reflected light from the interior of the plastic box because one projector pixel corresponds to several camera pixels in Fig. 6. Our experimental system detected the light transports with color mixtures between different channels in off-diagonal blocks $(\alpha \neq \beta)$ in extended-LTM. In Fig. 7, both meth-

\footnotetext{
* For the same reason, projector illumination proposed in 2) uses the square pattern of $3 \times 3$ pixels.
} 


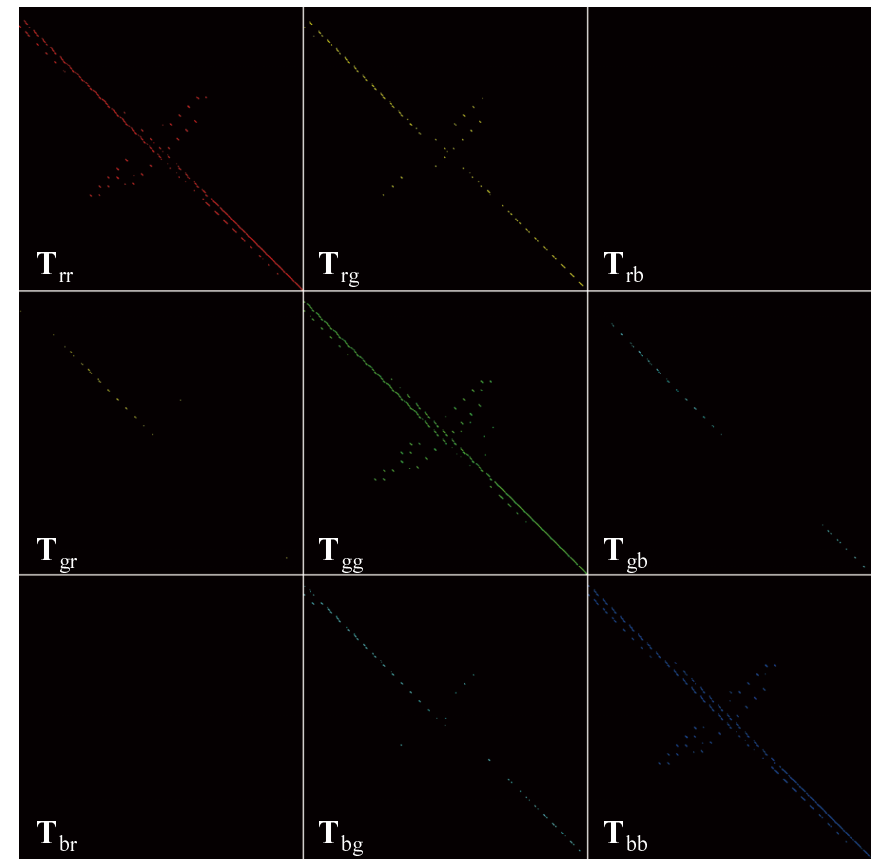

BFS

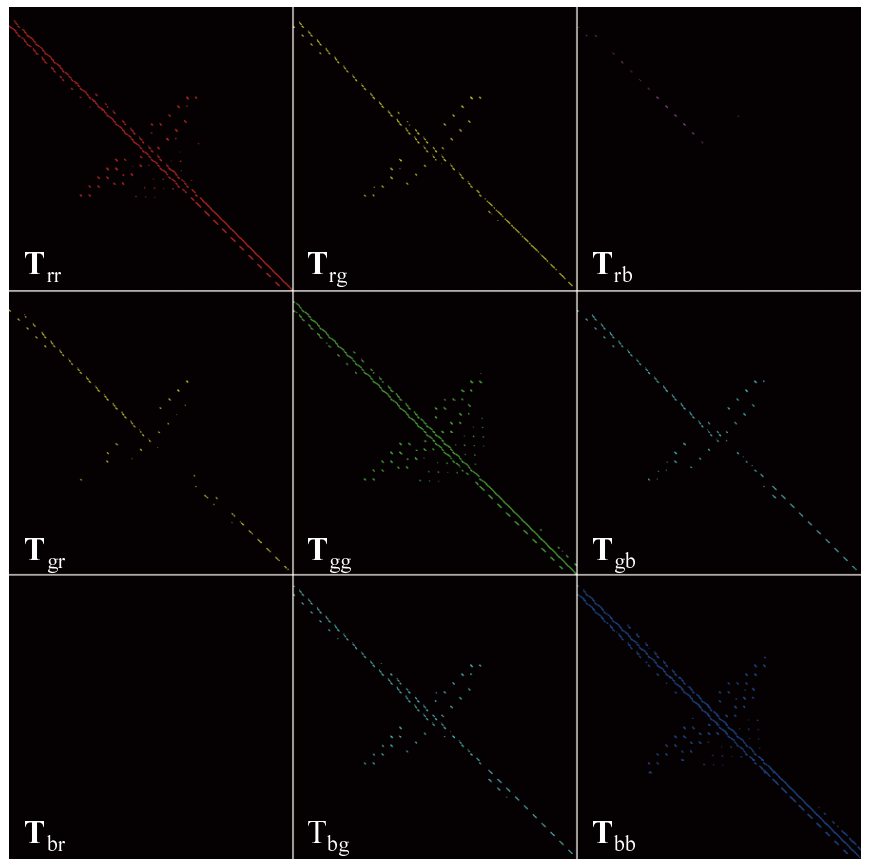

Our method

Fig. 6 Extended-LTM obtained from area A.

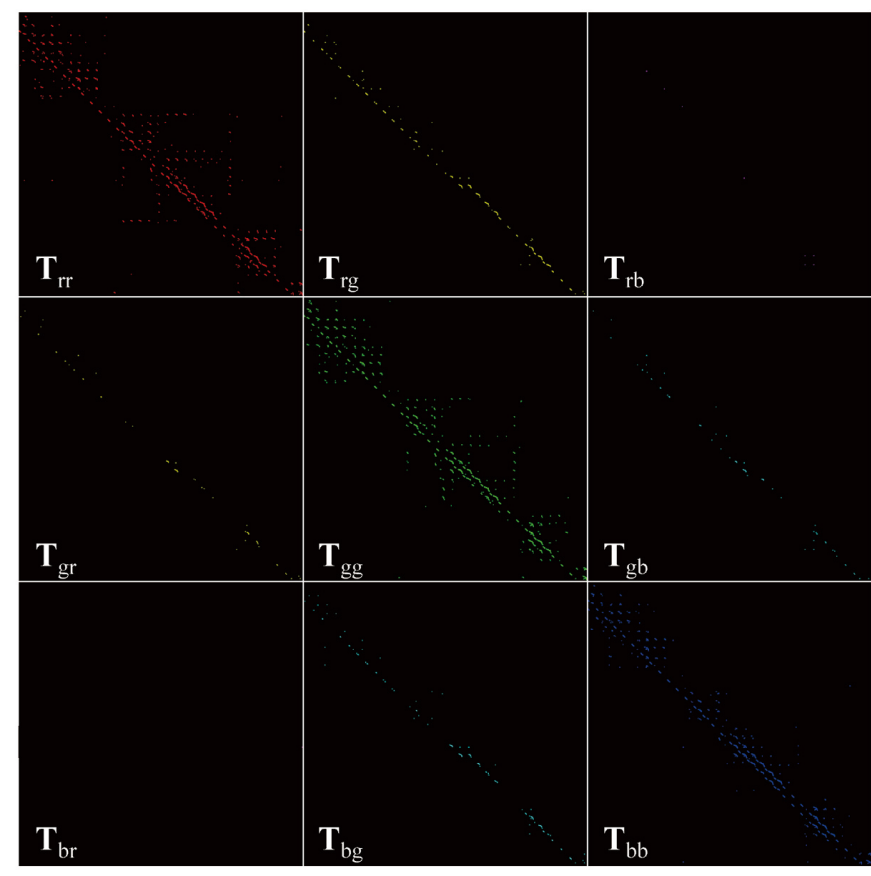

BFS

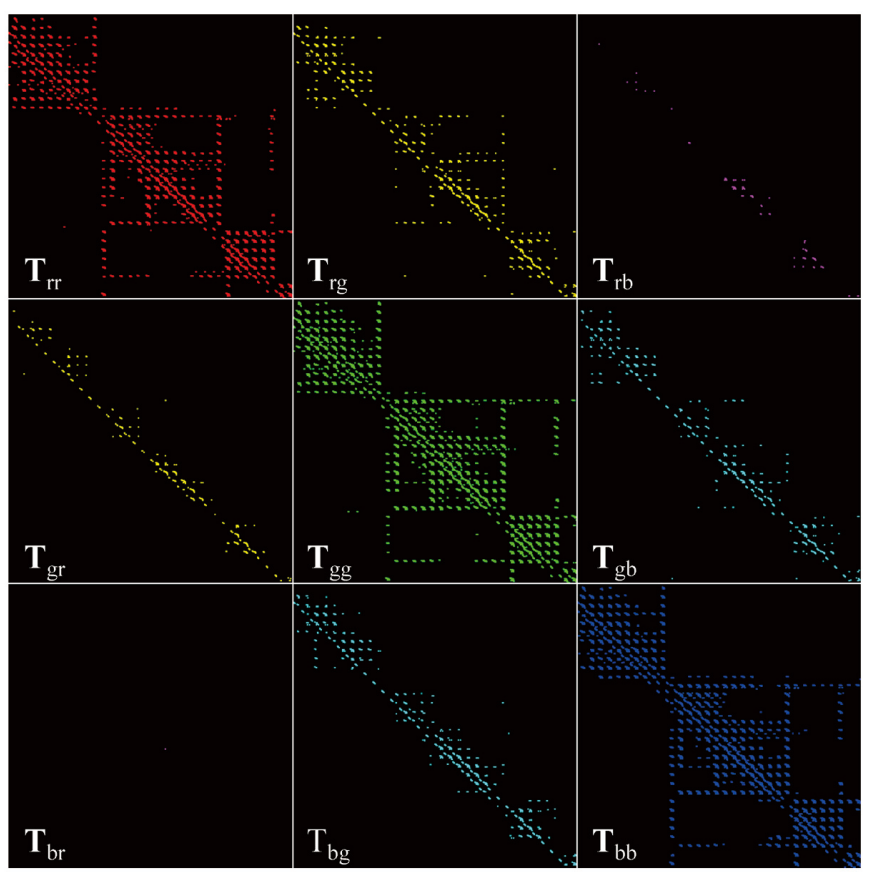

Our method

Fig. 7 Extended-LTM obtained from area B.

ods demonstrate that a projector pixel connects with many camera pixels, i.e. diffused reflection, and that multiple projector lights are transferred to a camera pixel. The detection of many direct and indirect reflected lights at a pixel region is an indicator of the phenomenon in which the surface of the plastic bottle frequently exhibited strong reflections. In contrast, Fig. 8 confirmed that light transports are dense in the diagonal components of LTM. These LTM show that many camera pixels are detected in the neighborhood of the point at which the projector light is directly reflected. The light transports that branch off the diagonal component are indicators of the lights refracted through the glass. Fig. 9 also shows that light transports were concentrated on the diagonal components. In other words, the metallic luster indicates that the 


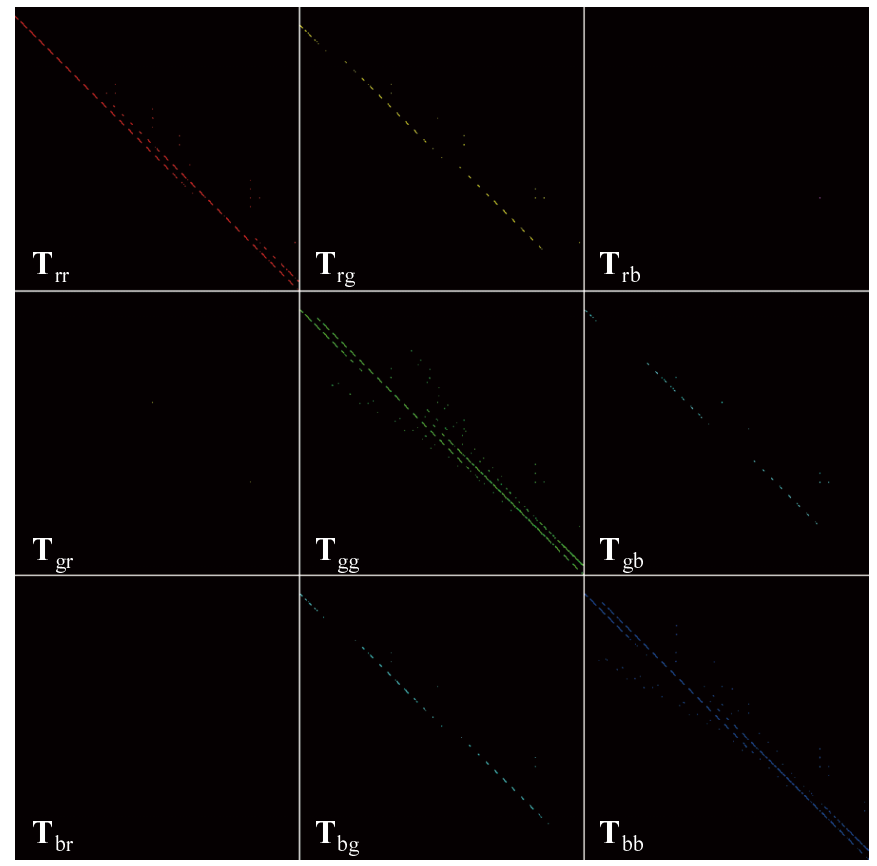

BFS

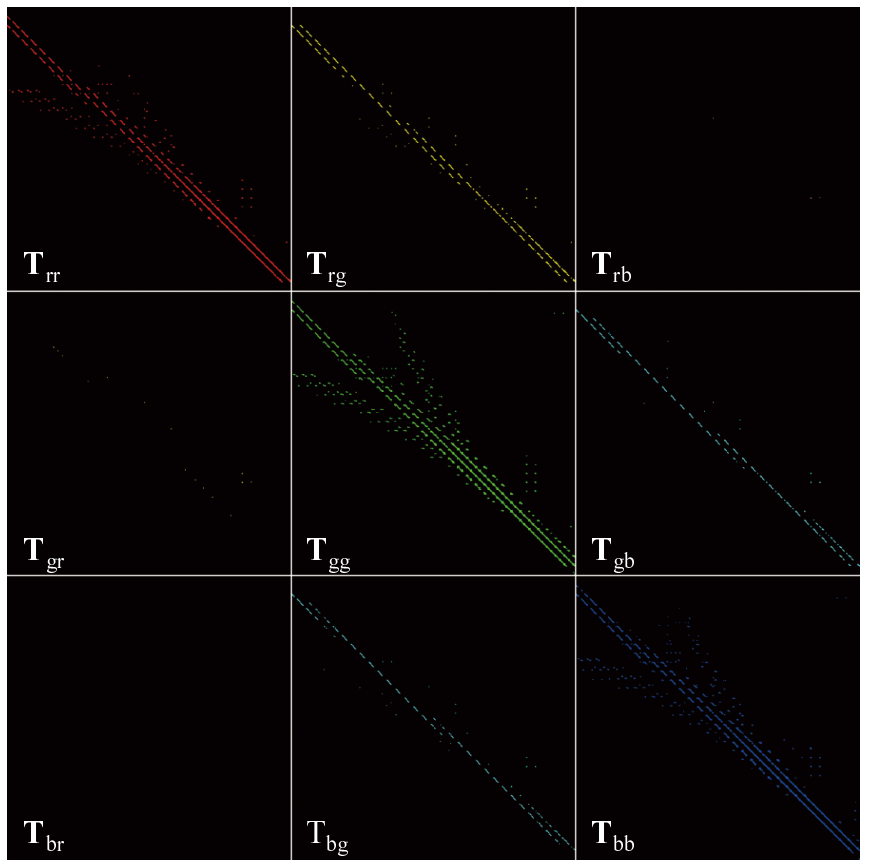

Our method

Fig. 8 Extended-LTM obtained from area C.

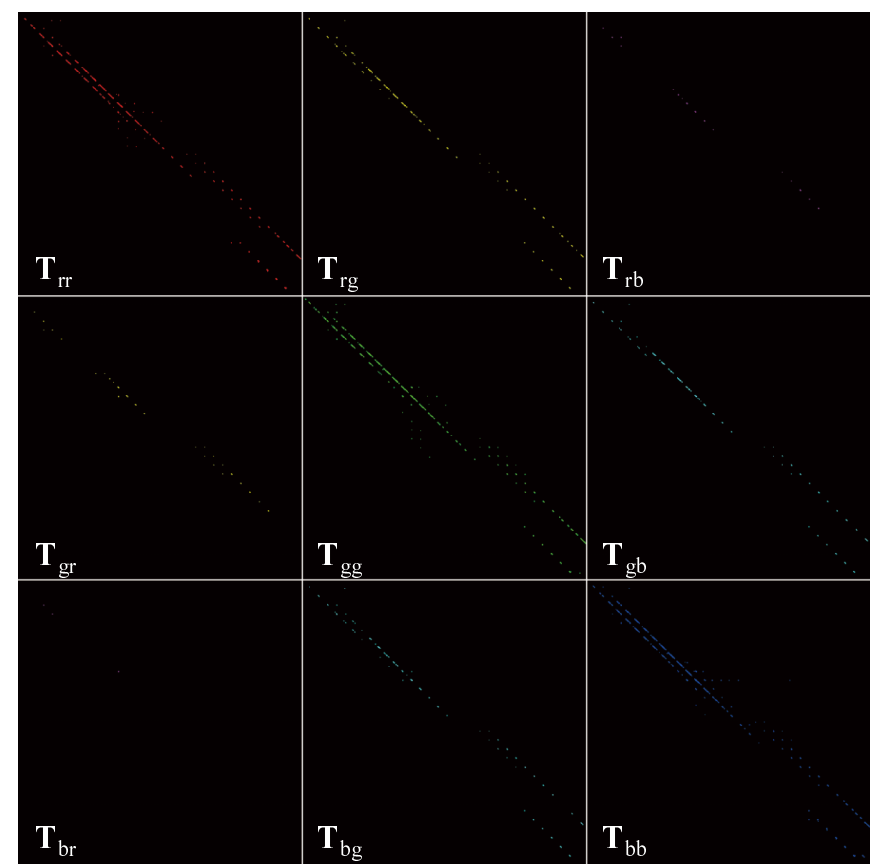

BFS

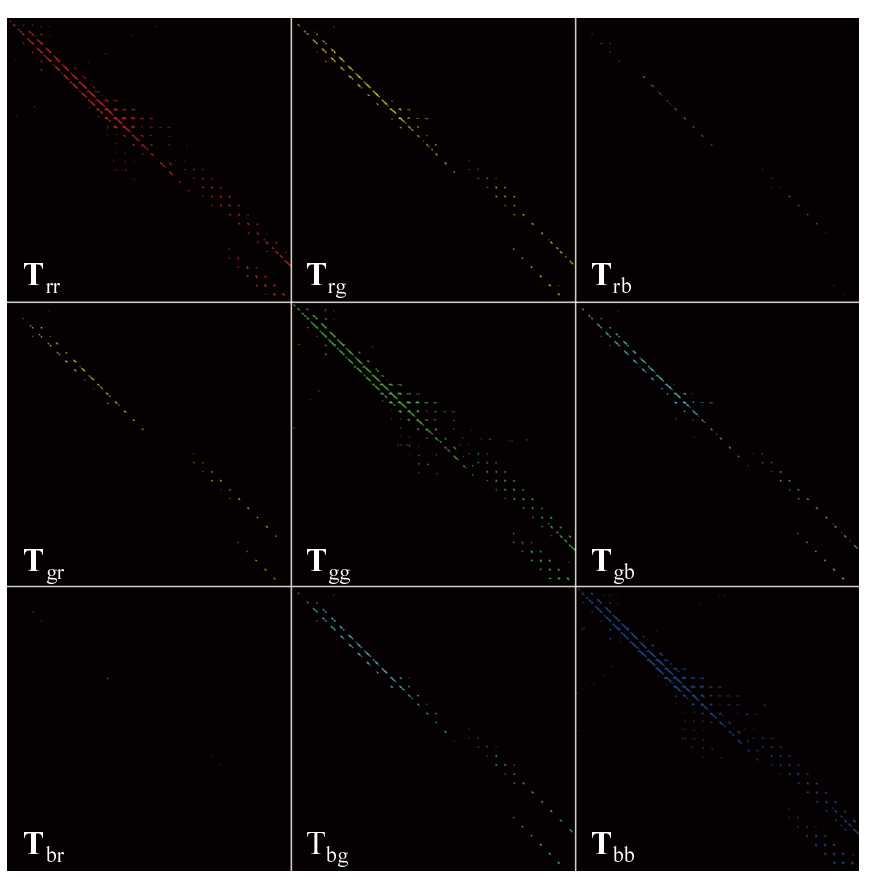

Our method

Fig. 9 Extended-LTM obtained from area D.

directivity of reflected light is high. As shown by the above results, the proposed method efficiently acquired direct and indirect light transports from just one sixteenth of the camera response images needed by BFS and that the extended-LTM acquired by our method has detailed light transports.

Next, we verify the accuracy of the extended-LTM acquired by BFS and our method. Given $N^{2}$-dimensional vectors $\mathbf{P}_{r}=\mathbf{P}_{g}=\mathbf{P}_{b}=[1,1, \cdots, 1]^{T}$, we reproduced original images by substituting the normalized $3 N^{2}$-dimensional projector vector $\left[\mathbf{P}_{r}^{T}, \mathbf{P}_{g}^{T}, \mathbf{P}_{b}^{T}\right]^{T}$ for Eq. (2). Fig. 10 shows the reproductions from the extended-LTM shown in Fig. 6, Fig. 7, Fig. 8, and Fig. 9. Fig. 10(a) shows actual images observed by our system, in which each 3D object was illuminated with white light. For reference, Fig. 10(c) shows images re- 


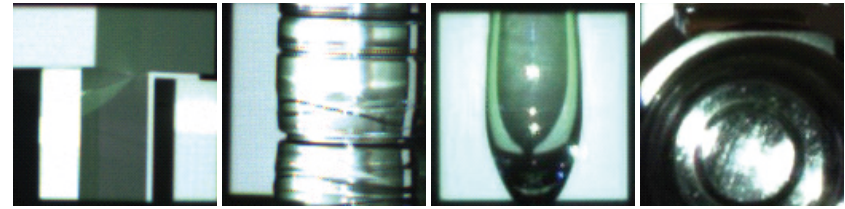

(a)
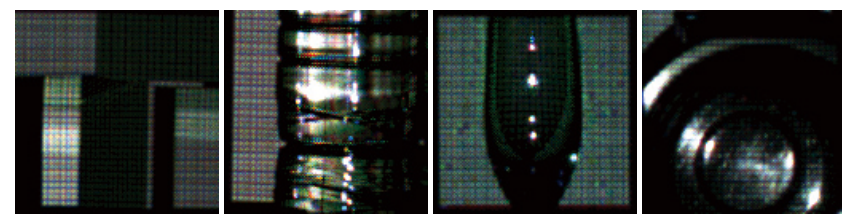

(b)

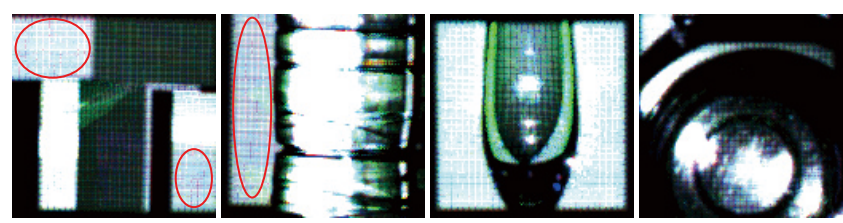

(c)

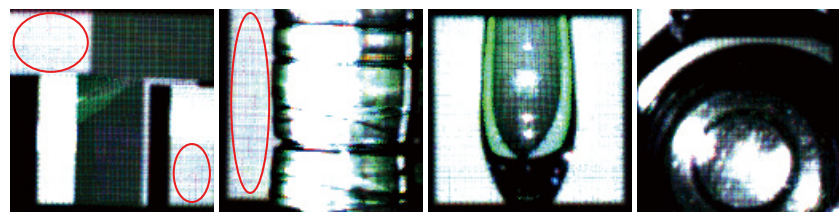

(d)

Fig. 10 Simulated image illuminated by white light. (a) Actual image. (b) BFS. (c) Our method without color mixtures. (d) Our method with color mixtures.

produced by using our method without color mixtures, i.e. $\mathbf{T}_{\alpha \beta}=\mathbf{0}, \alpha \neq \beta$, in the extended-LTM. See the pixel regions shown by the red oval in Fig. 10(c) and Fig. 10(d). Although the camera obtained almost white pixels at the regions in Fig. 10(a), our method without color mixtures reproduced slight different colors at the same regions. The results in Fig. 10(b) demonstrate that BFS yields markedly dark images. If we could customize the system for BFS by adequately adjusting optical parameters, e.g. shutter speed or aperture in the camera, BFS may yield more vivid images. In contrast, the proposed method with color mixtures accurately reproduces all images as compared to actual images. We determined that our system needs to consider the color mixtures between other color channels even though the mixture light transports are usually smaller than the pure light transports in extended-LTM. These results also demonstrate that the proposed method drastically reduces the number of camera response images needed to obtain the extended-LTM while still acquiring all light transports from complex 3D objects.

\section{3 Relighting}

In this subsection, we conduct a relighting test using extended-LTM. We used $512 \times 512$ projector pixels
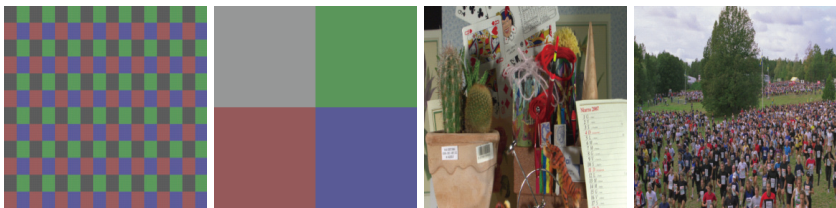

Fig. 11 Illumination images used in relighting test.

and $512 \times 512$ camera pixels; see area E in Fig. 5(a), and obtained camera response matrix $\hat{\mathbf{C}}$ and projector illumination matrix $\hat{\mathbf{P}}$ from the 768 camera response images output by the proposed method * The relighting using Eq. (2) demands working memory of $786,432 \times 786,432$ pixels in matrix $\mathbf{T}$. The memory limits of the computer prevented the relighting computation, even though we used the sparse matrix format in handling matrix T. Instead, by using Eqs. (14) and (15), the computation was feasible because matrix $\hat{\mathbf{C}}$ and matrix $\hat{\mathbf{P}}$ are compressed to $3 M^{2} \times 3 L^{2}$ and $3 N^{2} \times 3 L^{2}$, respectively. Given $3 N^{2}$-dimensional illumination vector $\mathbf{P}$, we calculate $3 L^{2}$-dimensional temporal illumination vector by $\mathbf{P}^{\prime}=\hat{\mathbf{P}}^{T} \mathbf{P}$, then obtain the $3 M^{2}$-dimensional camera response vector by calculating matrix product $\mathbf{C}=\hat{\mathbf{C}} \mathbf{P}^{\prime}$. Vector $\mathbf{C}$ yields a new image relighted by projector illumination $\mathbf{P}$.

Fig. 11 shows the illumination images used in this experiment. Fig. 12(a) shows the images captured by the camera under each illumination, as the comparison standard. Fig. 12(b) shows relighting results yielded by the proposed method. It realistically reproduced shading on the gypsum torso, which is very close to the real observation shown in Fig. 12(a). The plastic boxes demonstrate that the proposed method accurately yielded relighting with indirect reflection. Moreover, it faithfully reproduced lights reflected from the bottle's surface as well as lights refracted within the bottle. Our method also demonstrated that the illumination lights reflected from the side of the box overlap on the screen surface, e.g. pixel region shown by the red oval in Fig. 12. Namely, it accurately obtained light transports corresponding to direct and indirect reflection lights. Even if the projector illumination onto 3D objects is varied, the proposed method generates images that closely approximate what we would see in the real world. The method can promptly generate relighting results; it dispenses with acquisition of the updated LTM and the measurement of 3D structure.

* It took about 13 minutes to capture all camera images. If we employ BFS in this experiment, we would need to capture a total of 49,152 camera response images; this would take the system more than 13 hours. 

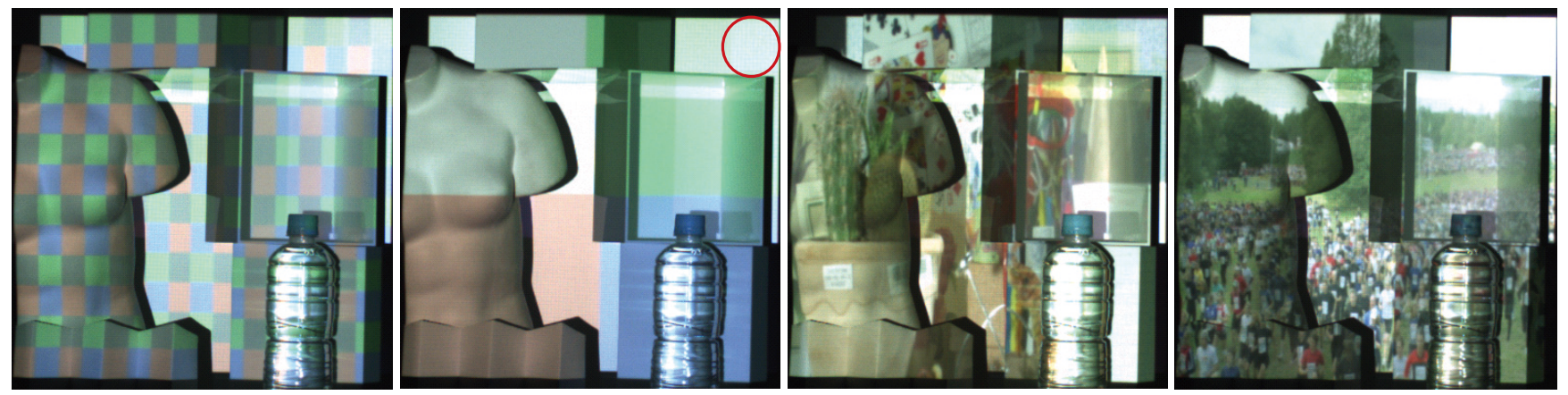

(a)
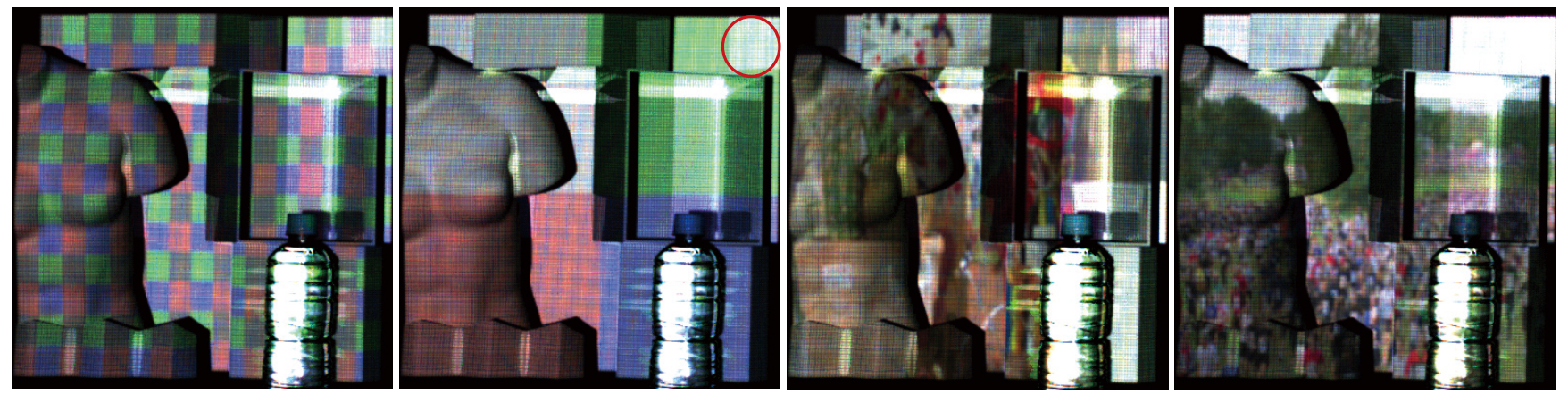

(b)

(b) Simulated images yielded by our method.
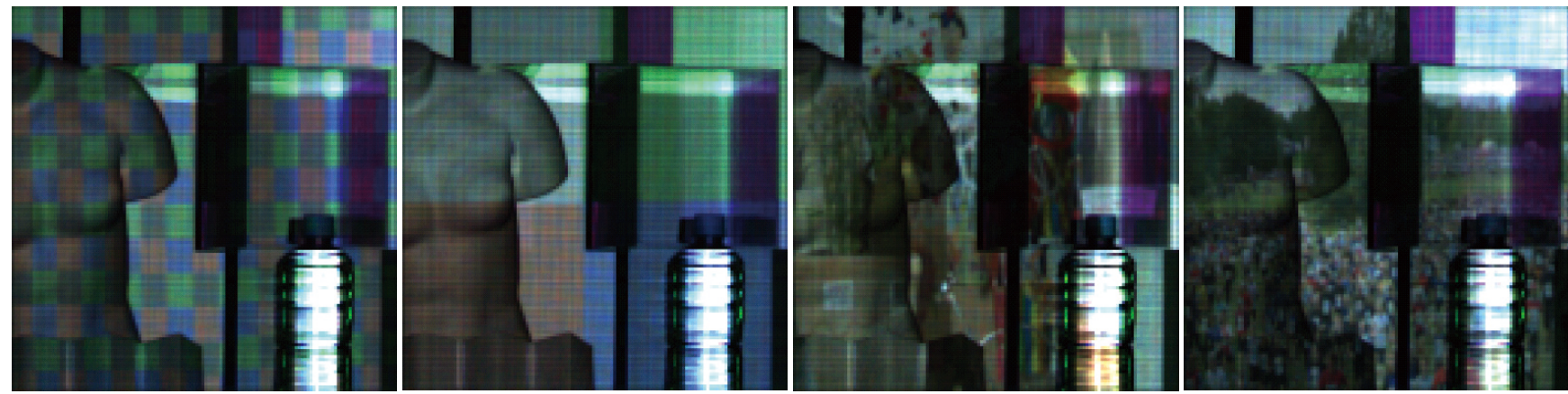

Fig. 13 Dual photography yielded from extended-LTM.

Fig. 13 shows dual photography generated by LTM transposition, $\left(\hat{\mathbf{P}} \hat{\mathbf{C}}^{T}\right) \mathbf{C}$, which creates a virtual image as observed from the projector's viewpoint ${ }^{2)}$, i.e. the camera illuminates the test images shown in Fig. 11. In Fig. 4(b), we locate a projector on the left side of a camera facing the 3D objects. By reversing the inputoutput relation between projector and camera, the projector virtually looks at the $3 \mathrm{D}$ objects from the righthand side viewpoint. See the plastic bottle with the shadowing in Fig. 12. Although the camera viewpoint in Fig. 12 places the shadow on the right side of the plastic bottle, the projector viewpoint in Fig. 13 places it on the bottle's left side. The gloss of water also shifts to the center region of the bottle in Fig. 13. This dual photography demonstrates that our method obtains accurate light transports between projector and camera pixels.

\section{Conclusion}

We proposed a practical method that efficiently acquires light transports from arbitrary objects by using vertical/horizontal-strip push broom-type projector illumination. We introduced a new LTM that handles the color mixtures between projector and camera pixels; our extension of the color-mixing matrix allows it to represent all direct and indirect reflected lights. We described the extended-LTM from a set of projector illuminations and camera responses. By utilizing all combinations of vertical-strip and horizontal-strip camera responses, our method detects, in common, the camera response, and obtains light transports between the overlapped projector pixels and the extracted camera responses.

In experiments, we acquired the extended-LTM from 
a group of complex 3D objects by using BFS and our method. We showed that our method efficiently obtained detailed direct and indirect reflected lights from these objects and accurately reproduced original images from the extended-LTM as compared with the actual camera images. Next, we conducted a relighting test based on the extended-LTM and confirmed that our method yields relighted images that closely approach camera observations. Dual photography based on the extended-LTM yielded good virtual images as observed from the projector's viewpoint. Our results demonstrated that the proposed method can significantly reduce the number of camera response images compared to BFS while acquiring a more accurate extended-LTM than BFS. Whenever users measure direct and indirect reflected lights by uncalibrated projector-camera systems, our method offers a high-speed alternative to BFS. As our method can acquire an extended-LTM that accurately represents $3 \mathrm{D}$ objects, relighting simulations based on the extended-LTM are useful when producing CG contents for projection mapping.

In the future, we will enhance our technique to achieve perceptually seamless, uniform, and highresolution video images. For projection mapping with higher presence, we need to compensate projector brightness according to visual performance. Although the inverse of LTM yields radiometric compensation, computing the inverse matrix will stress the working memory with the increased number of projector or camera pixels. To the best of our knowledge, no proposal can realize image displays by computing the inverse of LTM with high-resolution; existing solutions cannot realistically handle such enormous LTM. Instead, if we can discriminate between direct and indirect light transports in LTM, we can control flexibly projector illuminations that propagate projector pixels onto camera pixels based on the input-output relation of light transports. Future work includes separating direct and indirect reflected lights from LTM.

\section{Acknowledgment}

The constructive comments and advice from the editor and reviewers are gratefully acknowledged.

\section{References}

1) R. Ng, R. Ramamoorthi, and P. Hanrahan. All-Frequency Shadows Using Non-linear Wavelet Lighting Approximation. ACM Transactions on Graphics, Vol. 22, No. 3, pp. 376-381 (2003)

2) P. Sen, B. Chen, G. Garg, S. R. Marschner, M. Horowitz, M. Levoy, and H. P. A. Lensch. Dual Photography. ACM Transactions on Graphics, Vol. 24, No. 3, pp. 745-755 (2005)
3) S. M. Seitz, Y. Matsushita, and K. N. Kutulakos. A Theory of Inverse Light Transport. Proc. IEEE International Conference on Computer Vision, Vol. 2, pp. 1440-1447 (2005)

4) Y. Ding, J. Xiao, K-H. Tan, and J. Yu. Catadioptric Projectors. Proc. IEEE Conference on Computer Vision and Pattern Recognition, pp. 2528-2535 (2009)

5) T.-T. Ng, R. S. Pahwa, J. Bai, K.-H. Tan, and R. Ramamoorthi. From the Rendering Equation to Stratified Light Transport Inversion. International Journal of Computer Vision, Vol. 96, No. 2, pp. 235-251 (2012)

6) V. Masselus, P. Peers, P. Dutré, and Y. D. Willems. Relighting with 4D Incident Light Fields. ACM Transactions on Graphics, Vol. 22, No. 3, pp. 613-620 (2003)

7) G. Wetzstein and O. Bimber. Radiometric Compensation through Inverse Light Transport. Proc. Pacific Conference on Computer Graphics and Applications, pp. 391-399 (2007)

8) O. Nasu, S. Hiura, and K. Sato. Analysis of Light Transport based on the Separation of Direct and Indirect Components. Proc. IEEE International Workshop on Projector-Camera Systems (2007)

9) D. Mahajan, I. K. Shlizerman, R. Ramamoorthi, and P. Belhumeur. A Theory of Locally Low Dimensional Light Transport. ACM Transactions on Graphics, Vol. 26, No. 3 (2007)

10) J. Wang, Y. Dong, X. Tong, Z. Lin, and B. Guo. Kernel Nyström Method for Light Transport. ACM Transactions on Graphics, Vol. 28, No. 3 (2009)

11) M. O'Toole and K. N. Kutulakos. Optical Computing for Fast Light Transport Analysis. ACM Transactions on Graphics, Vol. 29, No. 6 (2010)

12) I. Miyagawa, Y. Sugaya, H. Arai, and M. Morimoto. An Iterative Compensation Approach Without Linearization of Projector Responses for Multiple-Projector System. IEEE Transactions on Image Processing, Vol. 23, No. 6, pp. 2676-2687 (2014)

13) S. K. Nayar, H. Peri, M. D. Grossberg, and P. N. Belhumeur. A Projection System with Radiometric Compensation for Screen Imperfections. Proc. IEEE International Workshop on Projector-Camera Systems (2003)

14) M. D. Grossberg, H. Peri, S. K. Nayar, and P. N. Belhumeur. Making One Object Look Like Another: Controlling Appearance Using a Projector-Camera System. Proc. IEEE Conference on Computer Vision and Pattern Recognition, Vol. 1, pp. 452-459 (2004)

15) K. Fujii, M. D. Grossberg, and S. K. Nayar. A Projector-Camera System with Real-Time Photometric Adaptation for Dynamic Environments. Proc. IEEE Conference on Computer Vision and Pattern Recognition, Vol. 1, pp. 814-821 (2005)

16) S. J. Koppal and S. G. Narasimhan. Shadow cameras: Reciprocal views from illumination masks. Proc. IEEE International Conference on Computer Vision, pp. 1211-1218 (2009)

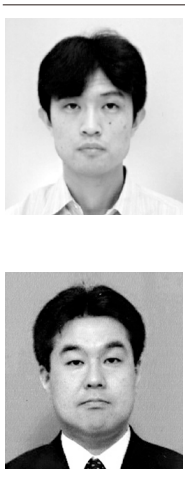

Isao Miyagawa received the B.E. degree in Electronics Engineering from Fukui University, Fukui, Japan, in 1991, and the Ph.D. degree in Engineering from Graduate School of Engineering of Fukui University, Fukui, Japan, in 2005. He joined NTT Corporation in 1991. He is currently pursuing a degree in computer vision. He is a member of IEEE, ITE, and a senior member of IEICE.

Hiroyuki Arai received the M.S. degree in Physics from Hokkaido University, Sapporo, Japan, in 1991, and the Ph.D. degree in Information Science from Hokkaido University, Sapporo, Japan, in 2009. He joined NTT Corporation in 1991. He is currently a senior research engineer of NTT Media Intelligence Laboratories. $\mathrm{He}$ is a member of the IEICE and ITE.

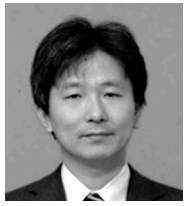

Yukinobu Taniguchi received the B.E. M.E., and Dr. Eng. degrees in mathematical engineering from the University of Tokyo in 1990, 1992, and 2002, respectively. He joined NTT Corporation in 1992. He is currently a senior research engineer, supervisor, of NTT Media Intelligence Laboratories. His research interests include image/video processing and multimedia applications. $\mathrm{He}$ is a member of the ACM, IEICE, IPSJ, and ITE. 